\title{
Melanoma proteomics unravels major differences related to mutational status
}

\author{
Lucía Trilla-Fuertes ${ }^{1 \#}$, Angelo Gámez-Pozo ${ }^{1,2 \#}$, Guillermo Prado-Vázquez ${ }^{2}$, Andrea Zapater- \\ Moros $^{2}$, Mariana Díaz-Almirón ${ }^{3}$, Claudia Fortes ${ }^{4}$, Rocío López-Vacas ${ }^{2}$, Iván Márquez-Rodas ${ }^{5,8}$, \\ Ainara Soria ${ }^{6}$, Juan Ángel Fresno Vara ${ }^{2,8}$ and Enrique Espinosa ${ }^{7,8,5}$ \\ ${ }^{1}$ Biomedica Molecular Medicine SL, Madrid, Spain \\ ${ }^{2}$ Molecular Oncology \& Pathology Lab, Institute of Medical and Molecular Genetics-INGEMM, \\ Hospital Universitario La Paz-IdiPAZ, Madrid, Spain \\ ${ }^{3}$ Biostatistics Unit, Hospital Universitario La Paz-IdiPAZ, Madrid, Spain \\ ${ }^{4}$ Functional Genomics Center Zurich, University of Zurich/ETH Zurich, Zurich, Switzerland \\ ${ }^{5}$ Servicio de Oncología Médica, Hospital Universitario Gregorio Marañón, Madrid, Spain \\ ${ }^{6}$ Servicio de Oncología Médica, Hospital Universitario Ramón y Cajal, Madrid, Spain \\ ${ }^{7}$ Servicio de Oncología Médica, Hospital Universitario La Paz-IdiPAZ, Madrid, Spain \\ ${ }^{8} \mathrm{CIBERONC}$ \\ "These authors contributed equally to this work \\ ${ }^{\$}$ Corresponding Author
}

Running title: Melanoma proteomics unravels major differences related to mutational status

Corresponding Author: Enrique Espinosa Arranz. Servicio de Oncología Médica, Hospital Universitario La Paz. Paseo de la Castellana 261, 28046 Madrid, Spain.

eespinosa00@hotmail.com

Conflicts of Interest and Source of Funding: JAFV, EE and AG-P are shareholders in Biomedica Molecular Medicine SL. LT-F is an employee of Biomedica Molecular Medicine SL. The other authors declare no competing interests. LT-F is supported by the Spanish Economy and Competitiveness Ministry (DI-15-07614). This study was supported by Instituto de Salud Carlos III, Spanish Economy and Competitiveness Ministry, Spain and co-funded by the FEDER program, "Una forma de hacer Europa" (PI15/01310). The funders had no role in the study design, data collection and analysis, decision to publish or preparation of the manuscript. 


\begin{abstract}
The aim of the study was to explore the molecular differences between melanoma tumor subtypes, based on BRAF and NRAS mutational status. Fourteen formalin-fixed, paraffinembedded melanoma samples were analyzed using a high-throughput proteomics approach, coupled with probabilistic graphical models and Flux Balance Analysis, to characterize these differences. Proteomics analyses showed differences in expression of proteins related with fatty acid metabolism, melanogenesis and extracellular space between BRAF mutated and BRAF non-mutated melanoma tumors. Additionally, probabilistic graphical models showed differences between melanoma subgroups at biological processes such as melanogenesis or metabolism. On the other hand, Flux Balance Analysis predicts a higher tumor growth rate in BRAF mutated melanoma samples. In conclusion, differential biological processes between melanomas showing a specific mutational status can be detected using combined proteomics and computational approaches.
\end{abstract}

\title{
Keywords:
}

Proteomics, personalized medicine, computational analysis, melanoma, flux balance analysis, probabilistic graphical models 


\section{Introduction}

Melanoma is the most lethal cutaneous cancer, with over 11,000-15,000 estimated deaths in the United States and Europe every year [1,2]. Better understanding of the molecular biology of this tumor has allowed the development of new effective drugs for the treatment of advanced disease, both in the fields of targeted therapies and immunotherapy [3]. However, as not all patients obtain a benefit from new drugs, further insight into the biology of melanoma is needed.

Gene signatures, genomic hybridization, whole-exome genome sequencing, microRNA analysis and other techniques have widely addressed the genomic landscape of melanoma, contributing to significant advances [4,5]. Given the heterogeneity of melanoma and the complex interaction of this tumor with the immune system, the need for combination of biomarkers assays has been recently proposed to properly analyze the disease [6].

Proteins determine cell phenotype, so proteomics analyses offer the possibility to measure the biologic outcome of cancer-related genomic abnormalities [7]. Mass spectrometry has become the method of choice to assess complex protein samples, and recent technological advances allow the identification of thousands of proteins from tissue amounts compatible with clinical routine. Therefore, proteomics may become a new source of molecular cancer markers offering complementary information to that provided by standard pathology and genomics. We recently demonstrated the feasibility of high-throughput label-free quantitative proteomics to analyze breast cancer from paraffin-embedded samples [8]. In the present study we sought to determine whether high-throughput proteomics combined with computational approaches, such as probabilistic graphical models and Flux Balance Analysis, are useful tools to explore functional differences between groups of melanoma tumors. 


\section{Methods}

Samples

Fourteen melanoma cancer patients were included in the study. FFPE samples were retrieved from Biobanks in IdiPAZ, Hospital Universitario Gregorio Marañón and Hospital Universitario Ramón y Cajal, all integrated in the Spanish Hospital Biobank Network (RetBioH; http://www.redbiobancos.es/). Patients provided informed consent. All experiments were performed in accordance with relevant guidelines and regulations. The histopathological features of each sample were reviewed by an experienced pathologist to confirm diagnosis and tumor content. Eligible samples had to include at least $50 \%$ of tumor cells. Approval from the Ethical Committees of Hospital Universitario La Paz was obtained for the conduct of the study.

Mass-spectrometry analysis protein identification and label-free quantification

Proteins were extracted from FFPE samples as previously described [9]. Peptides were desalted using self-packed C18 stage tips, dried and resolubilized with $15 \mu \mathrm{l}$ of $3 \%$ acetonitrile, $0.1 \%$ formic acid. Mass spectrometry analysis was performed on a QExactive mass spectrometer coupled to a nano EasyLC 1000 (Thermo Fisher Scientific). Solvent composition at the two channels was $0.1 \%$ formic acid for channel $A$ and $0.1 \%$ formic acid, $99.9 \%$ acetonitrile for channel B. For each sample $3 \mu \mathrm{L}$ of peptides were loaded on a commercial PepMapTM RSLC C18 Snail Column (75 $\mu \mathrm{m} \times 500 \mathrm{~mm}$, Thermo Fisher Scientific) and eluted at a flow rate of $300 \mathrm{~nL} / \mathrm{min}$ by a gradient from 2 to $30 \% \mathrm{~B}$ in $85 \mathrm{~min}, 47 \% \mathrm{~B}$ in $4 \mathrm{~min}$ and $98 \% \mathrm{~B}$ in 4 min. Samples were acquired in a randomized order. The mass spectrometer was operated in data-dependent mode (DDA), acquiring a full-scan MS spectra $(300-1700 \mathrm{~m} / \mathrm{z})$ at a resolution of 70000 at $200 \mathrm{~m} / \mathrm{z}$ after accumulation to a target value of 3000000, followed by HCD (higherenergy collision dissociation) fragmentation on the twelve most intense signals per cycle. HCD spectra were acquired at a resolution of 35000 using normalized collision energy of 25 and a maximum injection time of 120 ms. The automatic gain control (AGC) was set to 50000 ions. Charge state screening was enabled and singly and unassigned charge states were rejected. Only precursors with intensity above 8300 were selected for MS/MS (2\% underfill ratio). Precursor masses previously selected for MS/MS measurement were excluded from further selection for $30 \mathrm{~s}$, and the exclusion window was set at $10 \mathrm{ppm}$. The samples were acquired using internal lock mass calibration on $\mathrm{m} / \mathrm{z} 371.1010$ and 445.1200.

The acquired raw MS data were processed by MaxQuant (version 1.5.2.8), followed by protein identification using the integrated Andromeda search engine. Spectra were searched against a forward Swiss Prot-human database, concatenated to a reversed decoyed fasta database and common protein contaminants (NCBI taxonomy ID9606, release date 2014-05-06).

Carbamidomethylation of cysteine was set as fixed modification, while methionine oxidation and $\mathrm{N}$-terminal protein acetylation were set as variable. Enzyme specificity was set to trypsin/P allowing a minimal peptide length of 7 amino acids and a maximum of two missed-cleavages. Precursor and fragment tolerance was set to $10 \mathrm{ppm}$ and $20 \mathrm{ppm}$, respectively for the initial search. The maximum false discovery rate (FDR) was set to 0.01 for peptides and 0.05 for proteins. Label free quantification was enabled and a 2 minutes window for match between runs was applied. The re-quantify option was selected. For protein abundance the intensity 
was used, corresponding to the sum of the precursor intensities of all identified peptides for the respective protein group.

Following MS workflow, identified protein groups were filtered by the presence of at least two unique peptides and detectable expression in at least $75 \%$ of the samples. Label-free quantification values from these proteins were used for subsequent analyses. Additionally, batch effects were removed using limma package [10] and R v 3.2.5 [11].

\section{Protein differential expression analyses}

Significance Analysis of Microarrays (SAM) was performed using MeV to find significant differences in protein expression among samples [12]. Protein expression patterns were also compared calculating delta values for each biomarker status against the rest of the tumor samples. Proteins showing a change in expression value higher than 1.5 or lower than -1.5 were selected.

\section{Probabilistic graphical model and activity measurements}

R v 3.2.5 [11] and grapHD package [13] were used to build a probabilistic graphical model as previously described $[14,15]$. The network was split into several branches and Gene Ontology analysis was used to assign a major function to each node. Activity measurements were then calculated by the mean expression of all the proteins related to the assigned node function.

\section{Flux Balance Analysis}

Flux Balance Analysis (FBA) was performed using COBRA Toolbox [16] and whole metabolism human reconstruction Recon 2 [17] both available for MATLAB. As an objective function, biomass reaction supplied by the Recon 2 was used as representative of tumor growth rate. Proteomics expression data was incorporated into the model as described in previous works [15]. Briefly, GPR rules were estimated using the sum for "ORs" expressions and minimum for "ANDs" expressions. Then, E-flux algorithm [18] was used to normalize the GPR values dividing by the maximum value in each tumor and incorporate protein expression data into the model.

\section{Statistical analyses}

GraphPad Prism v6 was used for statistical analyses, whereas Cytoscape was used for network analysis. Gene Ontology Analyses were performed in DAVID webtool selecting "Homo sapiens" background and GOTERM-FAT, Biocarta and KEGG databases. 


\section{Results}

\section{Patients and samples}

Primary melanoma samples coming from 14 patients with advanced disease were included. Samples were split into three groups according to mutational status: BRAF-mutant $(n=3)$, NRAS-mutant $(n=5)$ or double negative $(n=6)$. BRAF and NRAS mutations had been previously determined in local laboratories with standard polymerase chain reaction-based tests.

Mass-spectrometry analysis

FFPE melanoma tumor samples were analysed by mass-spectrometry. 4,006 protein groups were identified, of which 1,606 present at least two unique peptides and detectable expression in at least $75 \%$ of the samples. Label-free quantification values from these 1,606 proteins were used for subsequent analyses.

\section{Differential protein expression patterns between subtypes}

A Significance Analysis of Microarrays (SAM) was done to find differences among samples at the protein level. Seventeen proteins were found differentially expressed between BRAF mutated and BRAF wild type tumors (Figure 1).

In addition, delta values between BRAF-mutated and BRAF-wild type, and NRAS-mutated and NRAS-wild type tumors were calculated. Delta values higher than 1.5 or lower than -1.5 were used to perform gene ontology analyses as well. Proteins related with keratinization, epidermis development and cytoskeleton were underexpressed, whereas proteins involved in melanogenesis and extracellular space were overexpressed in BRAF-mutant as compared with BRAF-wild type samples. SAM and delta analyses did not find significant differences between NRAS-mutant and NRAS-wild type tumors.

\section{Probabilistic graphical model and node activity measurements}

A probabilistic graphical model was built using proteomics data without other a priori information. The resulting network was processed to build a functional structure, as described in previous works $[14,15]$. The resulting network was divided into thirteen branches, and gene ontology analyses were performed to establish functional structure. Finally, twelve principal functions were assigned to different branches and there was a branch to which no main function could be assigned (Figure 2).

Node activity measurements were calculated for each node using proteins related with the main assigned function and a comparison between BRAF-mutant, NRAS-mutant and doublenegative groups was performed. Although the limited number of samples did not allow seeing significant differences, some trends in functional activities were found. For instance, NRASmutant had a lower melanosome node activity than BRAF-mutant or double negative tumors. On the other hand, BRAF-mutant tumors had a higher metabolism node activity than NRASmutant or double negative (Figure 3).

Flux Balance Analysis 
Flux Balance Analysis is a computational approach to assess biochemical networks through the calculation of the flow of metabolites through this network. FBA can be used to calculate the growth rate of an organism or the rate of production of a given metabolite. Our model predicted that BRAF mutated tumors have a higher tumor growth rate than the two other subtypes (Figure 4). 


\section{Discussion}

In this study, proteomics coupled with probabilistic graphical models and flux balance analysis were used to characterize differences between melanoma biomarker subgroups in melanoma samples.

Mass-spectrometry workflow allowed the detection of 1,606 proteins with two unique peptides and detectable expression in at least $75 \%$ of the samples. Differences in fatty acid metabolism, cytoskeleton or keratinization were observed between BRAF-mutant and BRAFwild type tumors. Also, differences in functions such as melanogenesis or metabolism were shown between subgroups

SAM and gene ontology analysis found 17 proteins differentially expressed between BRAFmutant and the two other subgroups (NRAS-mutant and double-negative). These proteins are mainly involved in fatty acid metabolism: acyl-Co A dehydrogenases ACADM, ACAA2 and ACADVL. Another protein with a differential expression is succinate dehydrogenase complex flavoprotein subunit A (SDHA), which encodes a major catalytic subunit of succinateubiquinone reductase, a complex of the mitochondria chain, and it was previously related with melanogenesis process [19]. Histidine/aspartate (HD)- domain containing protein 1 (SAMHD1) is implicated in regulation of DNA replication and damage repair and it is proposed to have antiproliferative and tumor suppressive functions in many cancers [20]. Sorting nexin 2 (SNX2) is involved in membrane trafficking of growth factor receptors including epidermal growth factor receptor and c-Met [21]. Another protein differentially expressed is the coagulation factor XIII (F13A1) which it was previously associated with chemotherapy response in melanoma tumors [22]. Potassium channel tetradimerization domain containing 12 (KCTD12) inhibits proliferation in uveal melanoma cells [23]. SLC9A3R1 is involved in suppressing breast cancer cells proliferation [24]. Annexin A6 (ANXA6) acts as a tumor suppressor in skin cancer and it is involved in in the conversion of melanocytes to malignant melanomas [25]. Integrin subunit beta 2 (ITGB2) participates in cell adhesion as well as cell-surface mediated signalling and it is correlated with survival in other cancers such as renal or colorectal tumors [26,27]. It was previously described that metastatic melanoma tumors have a decreased expression of signal transducer and activator of transcription (STAT1) and it could be one of the mechanism by which melanoma can evade immune detection [28]. Finally, G protein subunit alpha i2 (GNAI2) contributes to melanoma cell growth [29].

Differential analyses did not show differences between NRAS-mutant and NRAS-wild type tumors, which are attributable to the small sample size. The present study was limited in this regard because it was designed just as a proof of principle that high-throughput proteomics can be used to study clinical samples of melanoma. Future studies with larger sample size will be needed to establish significant differences among subtypes. Interestingly, it seems that delta analyses and SAM provide complementary information about different protein expression patterns, because differential proteins provided by these two analyses were related to different biological processes.

On the other hand, a probabilistic graphical model was used to generate a network based in protein expression data. It is remarkable that, despite the low number of samples, the probabilistic graphical model clearly showed a functional structure. This type of analysis 
previously demonstrated its utility to characterize other tumor types such as bladder carcinoma or breast cancer and may complement the information provided by genomics [15]. On the other hand, the high growth rate in BRAF-mutant tumors predicted by FBA agrees with previous knowledge [30].

In summary, our study demonstrates proteomics and computational methods can be applied to the study of clinical melanoma samples. Our results suggest that subgroups defined by mutational status have major differences at protein and functional levels. 
bioRxiv preprint doi: https://doi.org/10.1101/198358; this version posted October 6,2017 . The copyright holder for this preprint (which was not certified by peer review) is the author/funder. All rights reserved. No reuse allowed without permission.

\section{Acknowledgements}

We want to particularly acknowledge the patients in this study for their participation and to IdiPAZ, as well as participating Biobanks. 


\section{References}

1. Ferlay J, Steliarova-Foucher E, Lortet-Tieulent J, Rosso S, Coebergh JW, Comber H, et al. Cancer incidence and mortality patterns in Europe: estimates for 40 countries in 2012. Eur J Cancer 2013; 49 (6):1374-1403.

2. Ferlay J, Soerjomataram I, Dikshit R, Eser S, Mathers C, Rebelo M, et al. Cancer incidence and mortality worldwide: sources, methods and major patterns in GLOBOCAN 2012. Int J Cancer 2015; 136 (5):E359-386.

3. Dummer R, Keilholz U, Committee EG. appendix 2: Cutaneous melanoma (2): eUpdate published online September 2016 (http://www.esmo.org/Guidelines/Melanoma). Ann Oncol 2016; 27 (suppl 5):v136-v137.

4. Lin WM, Fisher DE. Signaling and Immune Regulation in Melanoma Development and Responses to Therapy. Annu Rev Pathol 2017; 12:75-102.

5. Bauer J. The Molecular Revolution in Cutaneous Biology: Era of Cytogenetics and Copy Number Analysis. J Invest Dermatol 2017; 137 (5):e57-e59.

6. Blank CU, Haanen JB, Ribas A, Schumacher TN. CANCER IMMUNOLOGY. The "cancer immunogram". Science 2016; 352 (6286):658-660.

7. Ellis MJ, Gillette M, Carr SA, Paulovich AG, Smith RD, Rodland KK, et al. Connecting genomic alterations to cancer biology with proteomics: the $\mathrm{NCl}$ Clinical Proteomic Tumor Analysis Consortium. Cancer Discov 2013; 3 (10):1108-1112.

8. Gámez-Pozo A, Trilla-Fuertes L, Prado-Vázquez G, Chiva C, López-Vacas R, Nanni P, et al. Prediction of adjuvant chemotherapy response in triple negative breast cancer with discovery and targeted proteomics. PLoS One 2017; 12 (6):e0178296.

9. Gámez-Pozo A, Ferrer NI, Ciruelos E, López-Vacas R, Martínez FG, Espinosa E, et al. Shotgun proteomics of archival triple-negative breast cancer samples. Proteomics Clin Appl 2013; 7 (34):283-291.

10. Ritchie M, Phipson B, Wu D, Hu Y, Law C, Shi W, et al. limma powers differential expression analyses for RNA-sequencing and microarray studies. Nucleic Acid Research2015. p. e47.

11. R Core Team. R: A language and environment for statistical computing. Vienna,Austria. R Foundation for Stattistical Computing, 2013.

12. Saeed Al, Sharov V, White J, Li J, Liang W, Bhagabati N, et al. TM4: a free, open-source system for microarray data management and analysis. Biotechniques 2003; 34 (2):374-378. 13. Abreu G, Edwards D, Labouriau R. High-Dimensional Graphical Model Search with the gRapHD R Package Journal of Statistical Software2010. pp. 1-18.

14. Gámez-Pozo A, Berges-Soria J, Arevalillo JM, Nanni P, López-Vacas R, Navarro H, et al. Combined label-free quantitative proteomics and microRNA expression analysis of breast cancer unravel molecular differences with clinical implications. Cancer Res, 2015. pp. 22432253.

15. Gámez-Pozo A, Trilla-Fuertes L, Berges-Soria J, Selevsek N, López-Vacas R, Díaz-Almirón M, et al. Functional proteomics outlines the complexity of breast cancer molecular subtypes. Scientific Reports 2017; 7 (1):10100.

16. Schellenberger J, Que R, Fleming R, Thiele I, Orth J, Feist A, et al. Quantitative prediction of cellular metabolism with constraint-based models: the COBRA Toolbox v2.0. Nature Protocols, 2011. pp. 1290-1307.

17. Thiele I, Swainston N, Fleming RM, Hoppe A, Sahoo S, Aurich MK, et al. A communitydriven global reconstruction of human metabolism. Nat Biotechnol 2013; 31 (5):419-425.

18. Colijn C, Brandes A, Zucker J, Lun D, Weiner B, Farhat M, et al. Interpreting expression data with metabolic flux models: Predicting Mycobacterium tuberculosis mycolic acid production. PLOS Comput Bio, 2009.

19. Boulton SJ, Birch-Machin MA. Impact of hyperpigmentation on superoxide flux and melanoma cell metabolism at mitochondrial complex II. FASEB J 2015; 29 (1):346-353. 
20. Kohnken R, Kodigepalli KM, Wu L. Regulation of deoxynucleotide metabolism in cancer: novel mechanisms and therapeutic implications. Mol Cancer 2015; 14:176.

21. Ogi S, Fujita H, Kashihara M, Yamamoto C, Sonoda K, Okamoto I, et al. Sorting nexin 2mediated membrane trafficking of c-Met contributes to sensitivity of molecular-targeted drugs. Cancer Sci 2013; 104 (5):573-583.

22. Azimi A, Pernemalm M, Frostvik Stolt M, Hansson J, Lehtiö J, Egyházi Brage S, et al. Proteomics analysis of melanoma metastases: association between S100A13 expression and chemotherapy resistance. Br J Cancer 2014; 110 (10):2489-2495.

23. Luo L, Cui J, Feng Z, Li Y, Wang M, Cai Y, et al. Lentiviral-mediated overexpression of KCTD12 inhibits the proliferation of human uveal melanoma OCM-1 cells. Oncol Rep 2017; 37

(2):871-878.

24. Liu H, Ma Y, He HW, Wang JP, Jiang JD, Shao RG. SLC9A3R1 stimulates autophagy via BECN1 stabilization in breast cancer cells. Autophagy 2015; 11 (12):2323-2334.

25. Qi H, Liu S, Guo C, Wang J, Greenaway FT, Sun MZ. Role of annexin A6 in cancer. Oncol Lett 2015; 10 (4):1947-1952.

26. Boguslawska J, Kedzierska H, Poplawski P, Rybicka B, Tanski Z, Piekielko-Witkowska A. Expression of Genes Involved in Cellular Adhesion and Extracellular Matrix Remodeling Correlates with Poor Survival of Patients with Renal Cancer. J Urol 2016; 195 (6):1892-1902.

27. Cavalieri D, Dolara P, Mini E, Luceri C, Castagnini C, Toti S, et al. Analysis of gene expression profiles reveals novel correlations with the clinical course of colorectal cancer. Oncol Res 2007; $16(11): 535-548$.

28. Osborn JL, Greer SF. Metastatic melanoma cells evade immune detection by silencing STAT1. Int J Mol Sci 2015; 16 (2):4343-4361.

29. Hermouet S, Aznavoorian S, Spiegel AM. In vitro and in vivo growth inhibition of murine melanoma K-1735 cell by a dominant negative mutant alpha subunit of the Gi2 protein. Cell Signal 1996; 8 (3):159-166.

30. Wellbrock C, Rana S, Paterson H, Pickersgill H, Brummelkamp T, Marais R. Oncogenic BRAF regulates melanoma proliferation through the lineage specific factor MITF. PLoS One 2008; 3 (7):e2734. 


\section{Figure legends}

Figure 1: Significance Analysis of Microarrays between BRAF positive and negative tumors.

Figure 2: Probabilistic graphical model built using protein expression data from melanoma tumors.

Figure 3: Activity measurements calculated for each network functional node according biomarkers features.

Figure 4: FBA predicted growth rates. 


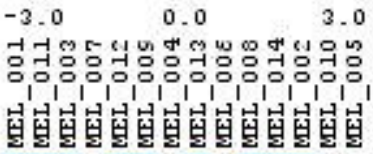

IRAS - 0

MRAS - 1

BRAF - 0

BRAF - 1

5
5
50
0
6
6

6

G)

$\infty$

$\infty$

m

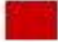

T

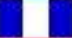

Б
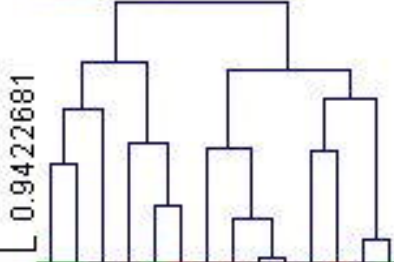

P 11310

P 42765

P 31040

P 14317

P04899

P42:24

P05107

P08133

P 630 7

014745 060749

P16402

Q9Y3Z3 Q96 CXa P00488

P49748 QSHOW 


\section{Splicing}

Extracellular matrix

Melanosome

Adhesion

mRNA y splicing

Protein processing

Mitochondria

Mitochondria 2

Ribosomes

Proteasome

Metabolism

Without function

Membrane

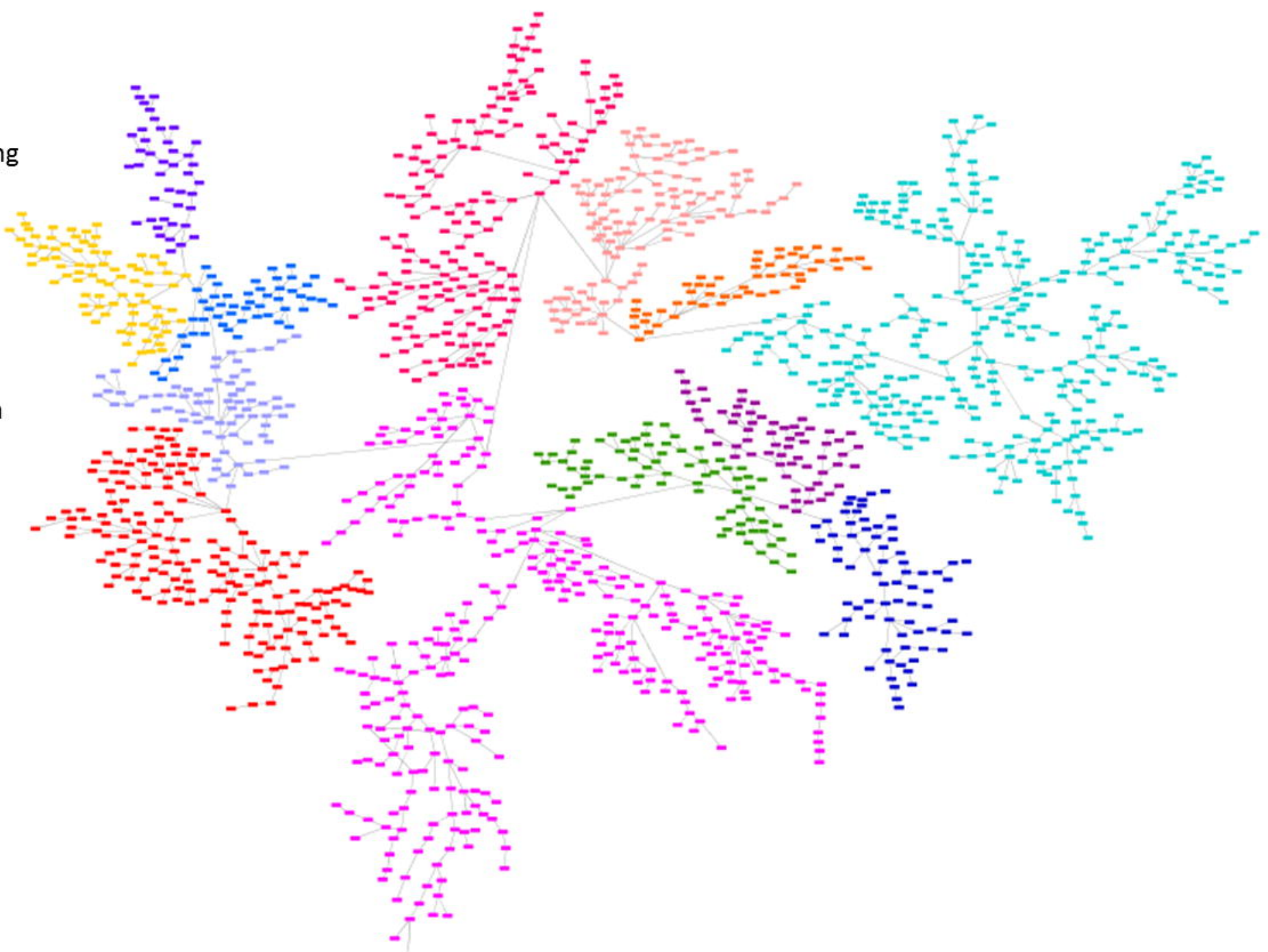


node 1 splicing

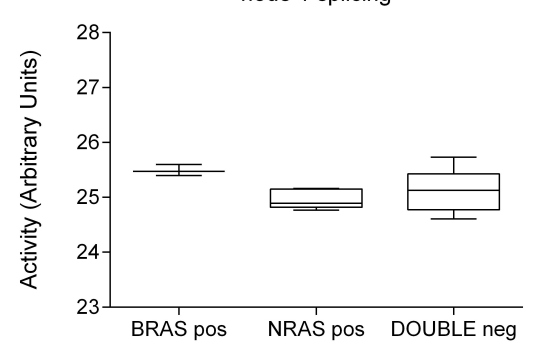

node 5 mRNA y splicing

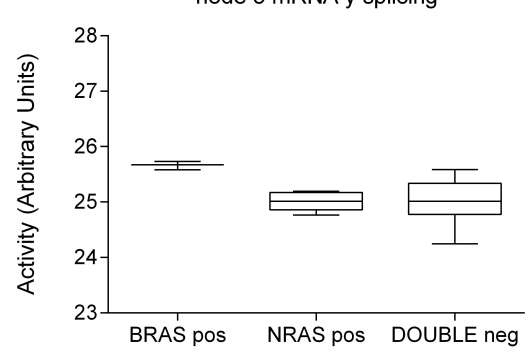

node 9 ribosomes

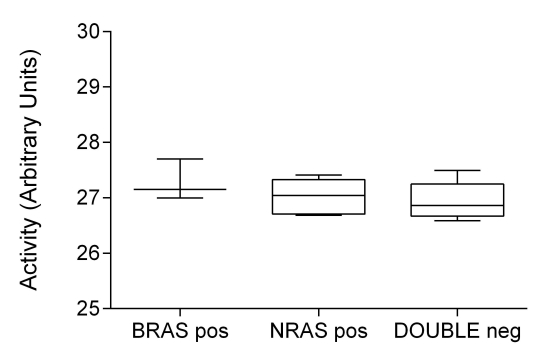

node 2 extracellular matrix

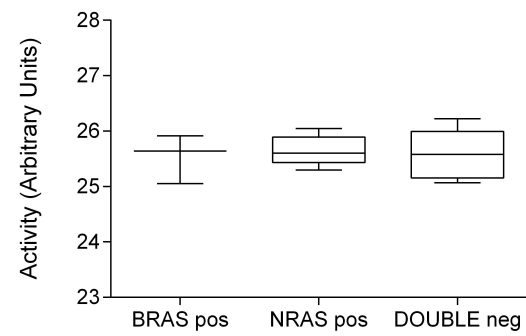

node 6 proteasome

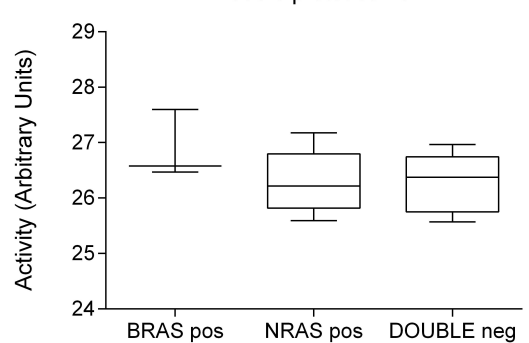

node 10 proteasome

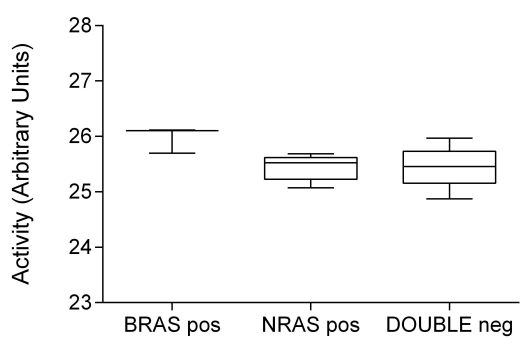

node 3 melanosome

node 4 adhesion
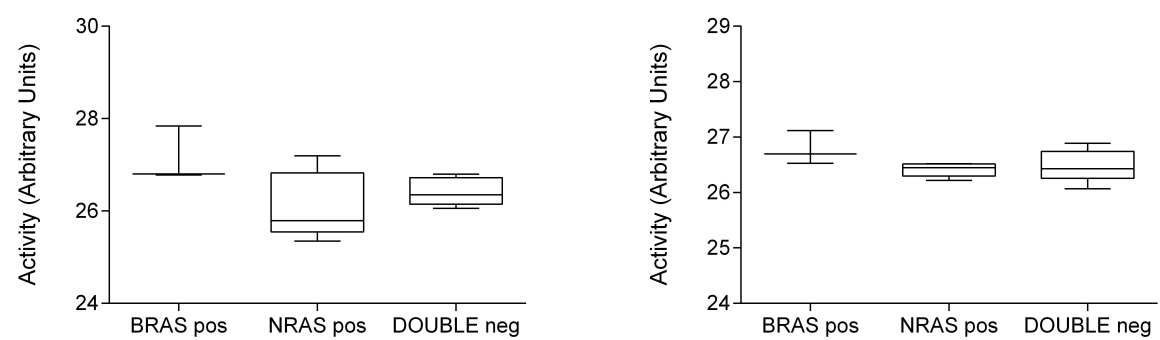

node 7 mitochondria
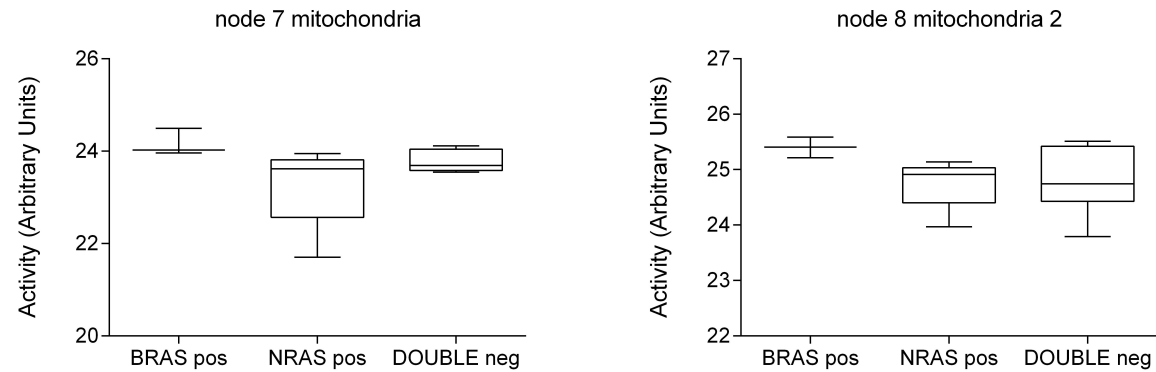

node 11 metabolism

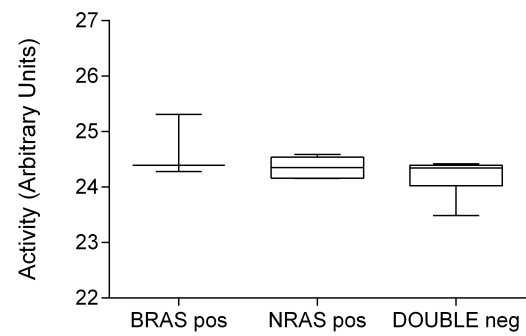

node 13 membrane

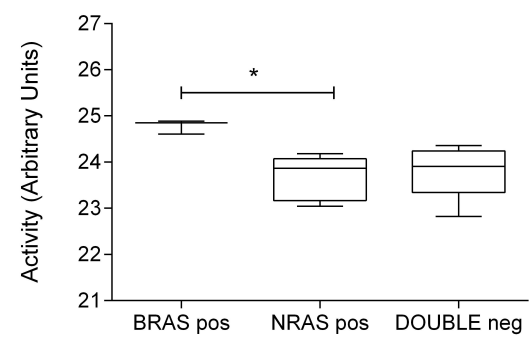




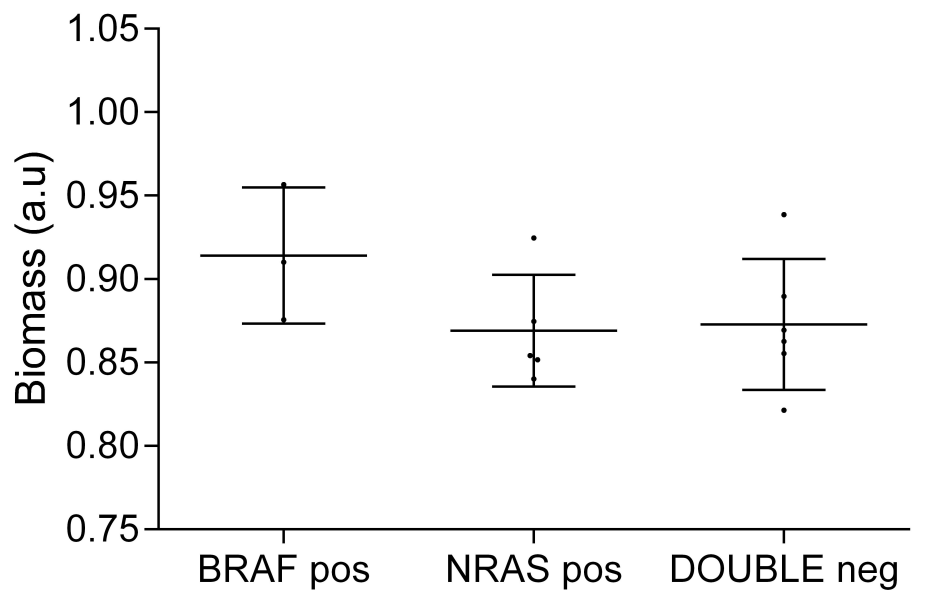

